\title{
Dieter Schwab Zum Selbstverständnis der historischen Rechtswissenschaft im Dritten Reich *
}

\section{l.}

Nachdem schon das Programm der NSDAP vom 25.2. 1920 (Punkt 19) den „Ersatz für das der materialistischen Weltordnung dienende römische Recht durch ein Deutsches Gemeinrecht« verlangt hatte, wurden seit der nationalsozialistischen Machrübernahme große Teile der Rechtswissenschaft von der Forderung nach völliger Umwälzung des gesamten Rechts (»Rechtserneuerung*) erfaßt. Die Feindseligkeit galt auch dem überkommenen Privatrecht, dessen Umgestaltung ein naiver Optimismus in kurzer Zeit leisten zu können giaubte. Man wird ermessen können, welche Bedeutung solche Tendenzen, gestützt auf das zur Gleichschaltung entschlossene Regime, für die beiden herkömmlichen Hauptzweige der rechtsgeschichtlichen Forschung haben konnte. Sah sich die Wissenschaft vom römischen Recht der Gefahr ausgesetzt, man werde ihr die Existenzberechtigung streitig machen, so konnte - so schien es - die »Germanistik *, die sich vorwiegend den germanischen Rechten und dem mitrelalterlich-deutschen Recht widmete, nach jahrzehntelanger Stellung an der Peripherie wieder in den Mittelpunkt rechtssystematischer Beachtlichkeit rüdken; sie konnte die Aussicht haben, ihre Bedeutung als historische Rechtswissenschaft mit dem Anspruch auf dogmatische Relevanz ihrer Arbeitsergebnisse zu erneuern.

Blickt man in die Zeitschrift des Bundes Nationalsozialistischer Deutscher Juristen, "Deutsches Recht « genannt, namentlich in die ersten Jahrgänge nach der Machtergreifung, so ermißt man die Aussichten, die sich den Germanisten darzubieten schienen. Fast alle Beiträge befassen sich mit der Rechtserneuerung aus dem Geist oder Wesen des deutschen Volkes und nicht wenige verweisen, auch bei der Behandlung einzelner Rechrsinstitute, auf germanisches oder deutsches Reche der Vergangenheit.

- Wir suchen heute das neue deutsche Volksrecht ... Ganz von vorn brauchen wir jedenfalls bei der Neugestaltung nicht zu beginnen, denn seit einem Jahrhundert etwa ist an der Wiedererwedkung gearbeiret worden, wie das Lebenswerk von Männern wie Jacob Grimm, Eichhorn und Otto v. Gierke erkennen läßr... Heute sind sie in ihrer auf das älreste deutsche Recht zurüdkgreifenden Arbeit gerechtfercigr: Endlich beginnt das deutsche Volk unter dem Einfluß nationalsozialistischer Erziehungsarbeit zu begreifen, welden Schatz cs an scinem alten Reche hat ${ }^{2}{ }^{2}$

- Der politische Soldat, wie ihn der Nationalsozialismus formt, steht in der Geschichte scines cigenen Volkes und stcht ihr nicht als unbetciligter Betrachter gegenüber. Das schließt zwar nicht aus, daß er bei der Betrachtung vergangener Zustände und Ordnungen des eigenen Volkes einen Abstand wahrt... der Betrachter ist der Geschichte seines eigenen Volkes doch zugleich willensmäßig zuinnerst verbunden... Ihm sind dieselben

- Vortrag, gehalten am I4. r. 1969 an der Justus-Liebig-Universität Giessen im Rahmen der Vortragsreihe * Nationalsozialismus und Rechta.

1 Hans Kod, Die Bauernweistümer und Recitsspridhwörter als Ausdrudk alten deutschen Rechtsempfindens und Volkstums, Deutsches Recht, Jg. 1934, S. 107. 
unvergängliahen Anlagen der Rasse überkommen, die schon in der Frühzcit gestaltend wirkten. Weil dicse Anlagen in Gesinnung und Wertungen zum Ausdruck kommen, darum können zuch in uns die gleiche Gesinnung und diesclben Wertungen wirken, die in den Rechten der germaniscien Stämme sich ausprägen ....ะ

Es bleibe nicht bei solchen Allgemeinheiten. Der Modellanspruch des VölkischRassisch-Geschichtlichen äußert sich vielfach in konkreten Nutzanwendungen. Rechtspolitische Vorschläge werden nicht selten mit dem Hinweis auf das alte Recht untermauert, wobci uns nicht die historische Haltbarkeit der Behauptungen interessiert, sondern die methodische Behandlung der vielfach zur historischen Utopie abgleitenden Geschichte. Uberschriften wie "Der Geist des deutschen und römischen Rechts in der Wirtschaft ${ }^{3}$ oder "Die Enneuerung unseres Verfahrensrechis aus dem germanischen Rechtsdenken « zeugen von der gewählten Methode. In dem zuletzr genannten Aufsatz heißt es:

- Eine Rechrsdogmatik, die dem Wesen des Führerstaats gerecht werden will, muß ... die alegermanischen Rechtsvorstellungen zugrundelegen. $\star^{5}$

Auf altgermanische Rechtsvorstellungen beruft sich ein Autor für seine unter dem Titel Der sittliche Ehebruch ${ }^{8}$ publizierte Forderung, das Kebsverhältnis als anerkanntes Rechtsverhältnis neben der Ehe zuzulassen. Die Kebsgemeinschaft, so meint er, ist «germanisches und deutsches Recht bis zur Zeit Martin Luthers gewesen. Sie war eine Rechtsform der Vergangenheit. Und Mut ist die Forderung der Zukunf**.

\section{II.}

Der Germanistik schien also nach einer verbreiteten Auffassung für die "Rechtserneuerung " eine führende Rolle zugedacht zu sein. Daß die Vertreter dieses Fachs oder ein großer Teil unter ihnen versuchen würden - und zwar ohne daß sie hätten Nationalsozialisten sein müssen -, einen solchen Part zu übernehmen, hatte, rückblickend gewerter, einige Wahrscheinlichkeit für sich. Entsprach doch die Forderung des Parteiprogramms nach Eliminierung des römischen im deutschen Recht den Vorstellungen, mit denen schon die Germanisten des I9. Jh. gegen das römische Recht zu Felde gezogen waren. Ein Blick auf die wissenschaftsgeschichtliche Situation mag dies verdeutlichen.

Ihre Größe und Blüte im Ig. Jahrhundert verdankt die rechtsgeschichtliche Forschung dem romantischen Geschichtsdenken, das bei Gründung der historischen Schule zu Beginn des Jahrhunderts das Forschungsziel bestimmte. Zwar bediente man sich der immer vollkommener behersschten historisch-kritischen Methode; als Ziel der Forschung wurde jedoch der Dienst am geltenden Recht proklamiert.?

2 Martin Bissse, Germanisches Bauernreche, Deursches Recht, Jg. I934, S. 497.

3 Ruciolf Bediert, Deutsches Reche, Jg. 1933, S. 81 ff.

4 Georg Lenz, Deutsdie Rechtswissenschaf, Jg. 1937, S. 338 ff.

5. 2. O., S. $338 / 339$.

- Rudolf Bedjert, Zeitschrift der Akademie für Deutsches Recht (ZAkDR), Jg. 1939, S. 446.

7 Dazu Karl von Amira, Ober Zweok und Mirtel der germanischen Rechtsgeschichte, München 1876; Walter Wilhelm, Zur juristischen Metnodeniehre im 19. Jh. Die Herkunft der Merhode Paul Labands aus der PrivatrecitswissenschaA, Frankfurt 1958, S. 17 ff.; Rudolf Gmür, Savigny und die Entwicklung der Rechtswissenschaft, Schriften der Gesellschaft zur Förderung der Westfülischen Wilhelmsuniversität, H. 49, Miünster 1962, S. 1s ff., 32 ff.; Erik Wolf, Große Rechtsdenker der deutschen Geistesgeschichte, 4. Aufl., Tübingen 1963, S. 486 ff.; Franz Wieacker, Privatrechesgeschidie der Neuzeit, 2. Aufl., Göttingen 1967, S. 381 f., insbes. 388 ff. 
Das historische Recht wurde als Ergebnis eines auf dem Volksgeist beruhenden Wachstumsprozesses aufgefaßt und so mit einem dogmatischen Anspruch bekleidet. Die "historische Methode" präsentierte sich folglich als ein Weg zur Rechtserkenntnis. Bekanntlich wurde die historische Methode nun aber sowohl am römischen, wie am germanischen und deutschen Recht geübt; diesem Umstand verdanken wir die Schulenbildung der Romanisten und Germanisten. Die Romanisten entfalteten in der Folgezeit das gewaltige System der Pandektenwissenschaft als umfassende Privatrechtsordnung. Die Germanisten versuchten demgegenüber, aus ihrem verstreut liegenden historischen Material, wenn auch zum Teil mit den begrifflichen Mitteln des römischen Rechts, ein System des genuin deutschen Privatrechts aufzurichten. ${ }^{8}$ Eben wegen des dogmatischen Anspruchs dieser historischen Rechtswissenschaften verwandelte sich die methodische Nachbarschaft sehr bald in eine erbirterte Feindschaft. Den letzten Höhepunkt der Auseinandersetzung bildete der Kampf um die Gestalt des Bürgerlichen Gesetzbuches. Der Ausgang des Streits ist bekannt: Das BGB erhielt durch die Pandektenwissenschaft seine bestimmende Form; germanistische Einsprüche, insbesondere durch Otto von Gierke, gegen den ersten Entwurf von 1887 fübrten zu keiner Revision des Konzepes im Ganzen. ${ }^{9}$

Spätestens mit dem Inkraftreten des BGB am I. Jan. I 900 war das Ende der * historischen Rechtswissenschaft « im bisherigen Verstande, und zwar nicht nur des germanistischen $Z$ weigs, eingeleitet. Eine auf Lückenlosigkeit bedachte gesetzliche Privatrechrsordnung, die den Geltungsanspruch auf einen ihr beigegebenen Geltungswillen stützt, läßt das historische Recht notwendig zur Vorgeschichte herabsinken. Die historische Rechtswissenschaft wird zur - auf das Phänomen des Rechts bezogenen - Geschichtswissenschaft. Mit dem dogmatischen Anspruch verior das geschichtliche Recht auch das Interesse des praktischen Juristen und nur deshalb, weil das Studium eben nicht einer primär praktischen Ausbildung dienen sollte, behielten die rechtshistorischen Vorlesungen, wenn auch von den Studenten häufig gemieden, cinen sehr brciten Platz in den Lehrplänen.

Eine neue Selbstbestimmung der rechtsgeschichtlichen Disziplinen war daher unerläßlich. Sie hatte freilich schon vor dem Inkraftereten des BGB begonnen. Für den Bercich der Germanistik ist die Münchner Antrittsrede Karl von Amiras (r 876) von besonderer Bedeurung. ${ }^{10}$ Von Amira forderte die Abkehr von der dogmatisch orientierten deutschen Rechtsgeschichte zugunsten einer als Teil der Kulturgeschichte begriffenen germanischen Rechtsgeschichte, die das Recht aller germanischen Stämme einbeziehen und nach Methode und Ziel der Geschichtswissenschaft zugeordnet werden solite. Im Gefolge dieses Programms entstand eine von dem Anspruch gegenwärtiger Brauchbarkeit befreite rechtsgeschichtliche Altertumswissenschaft. Auch die Arbeiten aus dem Bereich des mittelalterlich-deutschen Rechts hatten häufig schon im 19. Jh. ihre dogmatischen Gegenwartsbezüge abgestreift" ; die Rechrsgeschichre setzte sich zum Ziel die Erforschung historischer Sachverhalte und Einrichtungen.

Das neue Selbstverständnis mag freilich auch noch nach 1900 zuweilen weniger

\footnotetext{
8 Wieadter, a. a. O., S. 404 ff. mit weiterführender Lit.; Wilhclm, a. a. O., S. 88 ff.

- Dazu neuerdings Werner Schubers, Die Entstehung der Vorshriften des BGB über Besitz und Eigentumsübertragung, Berlin 5966, S. 4 s f., S. 5 I.

10 Uber Zwedk und Mittel der germanischen Rechtsgeschichte, Mündhen I 876 , insbes. S. I I ff.

11 Zum wissenschaftsgeschidelidien Vorgang im Ganzen vgl. Wieader, a. a. O., S. +16 ff.; Wieadker betont zu Recht, daß sić die Methode der Germanistik schon früher und gleichmäßiger der Gesdridaswissenschaft angeschlossen habe, als dies bei den Romanisten der Fall gewesen sci (S. 422).
} 
aus methodischer Einsicht, denn aus einer gewissen Resignation heraus akzeptiert worden sein ${ }^{12}$. Nicht akzeptiert wurde sie indes von Otto von Gierke, der nicht nur den ersten BGB-Entwurf aus deutschrechtlicher Sicht bekämpfte, sondern der sich, auch noch als die Kodifikation bevorstand und noch nachdem sie in Geltung gesetze war, niche davon abhalten licß, ein großes System des Deutschen Privatrechts zu schreiben, von dem drei Bände in den Jahren 1895 bis 1917 ersdienen sind ${ }^{13}$. "Der Kampf für deutsches Recht«, heißt es im Vorwort des ersten Bandes, skann und wird nicht ruhen, so lange es ein deutsches Volk gibt«. In seiner Schrift Die soziale Aufgabe des Privatrechts umriß er den dogmatischen Anspruch des alten deutschen Rechts wie folgt:

„... das Recht, welches uns heute formt, ist das erneuerte, das wiedergeborene, das aus todesähnliciem Schlummer erwedkte deutsche Recht. Nicht das deuesche Recht in seiner mittelalterlichen Gewandung, aber das deutsche Rechr in seinem unsterblichen Gedankengehalt... Darum knüpfen wir, wenn wir cin wahrhaft zukunftsreiches Rechr schaffen, so oft über die Episode der Fremdherrschaft hinweg an unsere große germanische Vergangenheit an ${ }^{14}$

Die Hoffnung von Gierkes, trotz des Sieges der Pandektistik im BGB werde der Tag des »deutschen Rechts « noch kommen, war - trotz methodischer Selbstbescheidung in den einzelnen Forschungsvorhaben - auch in den zwanziger Jahren noch lebendig. So hielt beispielsweise der Rechtshistoriker Herbert Meyer am S. Juni 1929 in Göttingen eine Rektoratsrede, die übrigens nach der Machtergreifung im Text unverändert erneut gedrudkt werden konnte ${ }^{15}$. In dieser Rede übt Mcyer scharfe Kritik an der seit dem Sieg der Pandektenwissenschaft vollzogenen Rechrsentwidklung und fordert die Rückkehr zu einem nationalen und volkstümlichen Rechr. Mit dem Aufruf "Zurück zu Gierke «16 will er aus der Summe deutschrechtlicher Geschiche die Grundgedanken des nationalen Rechts durchgeführt wissen. Dabei nennt er allgemeine und konkrete Positionen: deutsches Recht muß objektive Friedensordnung sein, darf also nicht das subjektive Recht zum Ausgangspunkt haben; das Privatrecht darf nicht individualistisch, es muß sozial sein; das deutsche Recht fordert das Prinzip der Kundbarkeit des Rechts; im Schadensersatzrecht muß das Verschuldensprinzip dem *Veranlassungsprinzip « weichen, und dergleichen mehr ${ }^{17}$.

Die Forderung des Nationalsozialismus nach Herstellung eines vom »Fremdeinfluß * gereinigten Nationalrechts mußte - so sollte man meinen - die Vertreter

12 Zugleich wurde jedoch auch die Erleichterung gespürc, die sich aus dem Wegfall des dokrrinären $Z$ wanges ergab, $\nabla$ gl. Ruclolf Hübner, Grundzüge des deurschen Privarrechts, 4. Aufl. Leipzig-Erlangen r 922: - Wir brauchen uns heut nicit mehr abzumühen, die Positivitär des deutschen Privarrechics zu begründen. Denn nur in den wenig zahlreichen Fällen, wo die dem Landesherrn vorbehaltenen Materien ohne partikulargeserzliche Regelung geblieben sind, befinder sich das deutsche Privatrecht noch beut in der gleichen unsicheren Lage wie ehedem. Im übrigen aber hat es durch die Aufriátung der deutschen Rechtseinheit eine völlig veränderte Stellung erhalten. Unser bürgerliches Recht ist das heutige, positive deutsche Privatreche und die Wissenschaft des bürgerlichen Reches unsere heutige gemeinrechtliche positiv-dogmatische Wissenschalt. Die Wissenschaft des deutschen Privatrechts hat demgegenüber eine propädeurische Aufgabe*.

13 Deuesches Privatreche, Leipzig bzw. München und Leipzig, $1895-1917$.

11 Berlin 1899, hier nach Frankfurt/Main 1943, S. II f.

15 Erste Veröffentlichung in: Mitreilungen des Universitärsbundes Görtingen, Jg. Ix Heft $I$, Görringen 1929; zweite Veröftentlichung Weimar 1933.

16 Zweite Ausgabe, S. 31 .

17 S. 32 ff. Für den Neubau des Staatsrechts auf germanistischen Grundlagen Walther Merk, Dez germanische Staat, Langensalza 1927, insbes. S. I2 ff. Siehe auch Claudius Freibert von Sohreerin, Der Geist des altgermanischen Rechts, das Eindringen des fremden Rechts, und die neuerliche Wiedererstarkung germanischer Rechtsgrundsätze, in: Germanisdıe Wiedererscehung, lig. von H. Nollau, Heidelbers 1926, S. $205 \mathrm{ff}$. 
der deurschrechtlichen Forschung vor allem deshalb ansprechen, weil dic nach I933 einsetzende juristische Schlagwortprogrammatik ihre Begriffe sehr häufig aus der Germanistik und der nationalen Geschichtsanschauung des 19. Jahrhunderts entnommen hatre. Die, von der Rassenfrage abgesehen, verschwommene nationalsozialistische Ideologie stellt ein Konglomerat von offenen Begriffen wie »völkisch «, "arteigen «, »artfremd «, »überfremdet «, sindividualistisch «, »sozial « usw. dar, die vielfach anderen Denkmodellen und Anschaunngsweisen entnommen sind, bei denen die Begriffe aber - in anderem Denkzusammenhang stehend - ganz andere Sinngehalte und Stellenwerte aufweisen konnten. So vermochte das nationalsozialistische Programm auf sehr unterschiedliche Gruppen und Kräfte Eindruck zu machen. Da der Nationalsozialismus dabei auf stagnierende oder resignierende Strömungen spekulierte, ging er vielfach zu Recht von der Annahme aus, man werde angesichts dergleichen Wertungsbegriffe und der durch sie vermittclten Assoziationen gegenüber den spezifischen Charakter der Bewegung blind sein oder sein wollen.

In der betont nationalsozialistischen Literatur zur Rechtserneuerung kommen Wertungen zum Durchbruch, finden ferner Begriffe Anwendung, die vielfach germanistischen Vorstellungen und dem spezifisch nationalen Geschichrsbild des I9. Jh. entnommen sind. Das gilt vor allen von der These, daß die deutsche Kultur durch die Geltung rönischen Rechts überfremdet sei ${ }^{18}$. Demgemäß sah das seit 1933 im Schwange befindliche Geschichtsbild entweder schon in der Rezeption des römisch-kanonischen Rechts ${ }^{19}$ zu Ende des MA oder aber im Aufkommen der Pandektistik des 19. Jh..20 eine zu krassem Individualismus und Materialismus führende Fehlentwidklung. Die vorbildliche Daseinsform genuin deutschen oder germanischen Rechts konnte demgegenüber im vorwissenschaftlichen germanischen und deutschen Recht gefunden werden, sofern man nur irgend einer Rechtsordnung Modellcharakter zuzuweisen geneigt war. Neben das historische deutsche Recht treten als Leitbilder freilich gelegentlich noch das englische und die nordeuropäischen Rechte, da in diesen Rechrskreisen eine Rezeprion des römischen Rechts in complexu nicht stattgefunden hatte"1. Ferner nahm die nationalsozialistische Rechrswissenschaft die aite germanistische Forderung nach Volkstümlichkeit und Volksverbundenheit des Rechts auf22. Hier galt eine verbreitete Feindschaft insbesondere dem BGB, das man als Ausgeburt volksfremder Pandektistik verstand ${ }^{23}$. Nach der damals gängigen Vorstellung bieter das BGB ein für das Volk unverständliches, abstraktes, lebensfremdes Recht, durch das der Volksgenosse der Geheimwissenschaft eines gelehrten Juristenstandes ausgelie-

13 Näheres über die Germanistik des 19. Jh. bei Hans Thieme, Savigny und das Deutsche Recht, ZRG Germ. Abr. 80 (1963), S. II ff.

10 Carl Sdjmitt, Die geschichrliche Lage der deurschen Rechrswissenschaft, DJZ 1936, S. 17; ders., Aufgabe und Norwendigkeit des deurschen Rechtsstandes, Deutsches Reche 1936, S. 181; W. J. Hedemann, Der Gesamtbau des bürgerlichen Rechts, ZAkDR 1935 S. 43I; Manche - Deurschrechtier sahen in der Rezeprion indes weniger eine Aufnahme von römischen Institutionen als die eines neuen, nämlich wissenschaftlichen Denkstils, ohne daB das Reche selbst überfremder worden sei, vgl. Herbert Meyer, Rasse und Reche bei den Germanen und Indogermanen, Forschungen zum Deutschen Rechr, Bd. II I3, Weimar 1937, S. 3I.

20 Z. B. Carl Sommitt, a. a. O., S. I9.

21 Vgl. Walcljer Merk, Wachstum und Schüpiung im germanischen Reche, Festgabe für Erich Jung, Marburg 1937, S. 173; Karl Midjaelis, Wandlungen des deutschen Rechtsdenkens scir dem Eindringen des fremden Rechts, Berlin 1935. S. I8, s. aber S. 55.

2: Grundlegend die Schrift von Georg Beseler, Volksrecht und Juristenreche, Leipzig i 943 .

:3 Vgl. Frank, Deurscies Recht, Jg. 1936, S. 298; Michaelis, 2. a. O., S. 49 f., Sdblegelberger, Abschied vom BGB, Berlin I93-, S. to. Je länger sich freilich die Jurisprudenz mit den Vorbereitungen zur neucn Gesetzgebung beschäftigre, desto mehr wuchs die Anerkennung für dir Leistungen der Schöpfer des BGB, vgl. die interessante wissenschattsgeschichtliche Arbeit von Heinrich Lange, Die Entwidklung der Wissenschatt vom Bürgerlichen Recht seit 1933, Tübingen 1941 . S. 20. 
fert ist. Wie leicht konnte man demgegenüber das Ideal der germanischen oder mittelalterlich-deutschen Rechtskultur beschwören, in der das Recht niche von oben gesetzt, sondern im Volk und durch es selbst gefunden worden sei, in der das Recht also nicht über dem Volk, sondern in ihm gelebt habe ${ }^{24}$. Besonderer Verachtung erfreute sich, nidht nur von den rechtstheoretisch konzipierten Positionen des konkreten Ordnungsdenkens her ${ }^{25}$, der Allgemeine Teil des BGB, den man als den Gipfel pandektistischer Konstruktionsjurisprudenz empfand ${ }^{2 n}$. In diesem Zusammenhang verband sich freilich mit der Forderung nach Volkstümlichkeit eine dem Nationalsozialismus eigene Tendenz zum Irrationalismus"7. Die Abreigung gegen das *Abstrakte«, Vergeistigte, gegen reflektierte Grundlirien der Rechtsordnung ging soweit, daß man verschiedentlich an die Imitation des englischen case law dachte ${ }^{28}$, eine Bestrebung, die aus begreiflichen Gründen keine Verwirklichungschance besaß. Auch hier konnte aber das völlig unsystematische, stets auf typische Sachverhalte bezogene mittelalterliche Recht zum Kontrast genommen werden. Im Zusammenhang mit der Abstraktheit des BGB wurde vielfach seine Sprache einem Verdikt unterzogen. Einfach, jedermann verständlich sollte die Rechtssprache sein, wie dies eben in der mittelalterlich-deutschen Rechtsordnung der Fall gewesen ist, deren Sinnfälligkeit - man denke nur an die Paarformeln - zu den Eigenarten vorwissenschaftlichen Rechrs gehört. Eine besondere Rolle für den Nationalsozialismus spielte bekanntich die Ubernahme und Mychologisicrung des Treubegriffs.

Schließlich sei eine letzte Ubereinstimmung germanistischer Tradition und nationalsozialistischer Schlagwortideologie erwähnt. Sie betrifft den Gedanken der sozialen Natur der subjektiven Rechte. Der nationalsozialistische Vorwurf des Individualismus und Materialismus traf das römisch geprägte Privatrecht, das als System auf die Willensherrschaft des privatautonomen Individuums gegründer war ${ }^{29}$. Die Germanisten hatten im Zuge der in der zweiten Hälfte des 19. Jahrhunderts rasch fortschreitenden Idee von der Sozialgebundenheir des Eigentums und anderer Privatrechte auch in diesem Punkt Kritik am Pandektenrecht geübt. Otto von Gierke versuchte in seiner Auseinandersetzung mit dem BGB demgegenüber das deutsche Privatrecht als ein von seiner Struktur her sozialbezogenes zu erweisen ${ }^{30}$. Von Gierkes Auffassung steht deutlich im Gefolge der Kritik, welche ökonomische Lehren des 19. Jahrhunderts am absoluten Eigentumsbegriff der Pandektistik geleistet hatten. Freilich stützr der Germanist die Aussage von der notwendig öffentlichrechtlichen Relevanz wichtiger Eigentumsarten nicht auf die Einsicht in wirtschaftliche Zusammenhänge; vielmehr geht er von der Beschaffenheit des nationalen Rechts aus. Gleichwohl steht von Gierke sichtlich unter dem Einfluß des neuen Sozialgedankens: „... in unserem öffentichen Recht muß ein Hauch des naturrechtichen Freiheitsraumes wehen und unser Privatrecht muß ein Tropfen sozialen Oles durchsickern «.31 Der

24 Vgl. F. Cornelius, Der Rechtsbegriff des Deutschen Rechts, Deutsches Recht, Jg. I934, S. I03.

95 Vol. dazu Peter Thoss, Der Weg des subjektiven Rechts in die gliedschattliche Bindung, Zum Verhältris von Nationalsozialismus und Privatrecht, S. 39 ff.

* Siehe z. B. Soblegellerger, 2. 2. O., S. 21. Eine Auseinandersetzung mit diesem gängigen Ureil finder sich bei Philipp Heek, Der Allgemeine Teil des Privatreches, ArchCivPrax 146 (1941), S. I ff.

$=\mathrm{V}_{\mathrm{gl}}$. Bernd Rüthers, Die unbegrenzre Auslegung, Tübingen 1968, S. $133 \mathrm{ff}$.

sa Zu diesen Bestrebungen Heinrioh Lange, Lage und Aufgabe der deutschen Privatrecteswissenschaft, Tübingen 1937 , S. Is ff.

- Hitler verwarf in einer Rede vom 30. Jan. 1937 die Auffassung, daß das Reche im wesentlichen berufen sei, den Shutz des Individuums in der Person und in seinem Eizentum zu übernehmen * (aus Z $A k D R$ 1937, 97).

9 Die soziale Aufgabe des Privatrechts, S. 6 und passim.

I a. а. O., S. 10. 
Nationalsozialismus bediente sich freilich des Gedankens der Sozialgebundenheir der Privatrechte mit einer ganz anderen Sinngebung. Sozialgebundenheit wurde zur totalen Eingliederung. Immerhin konnte auch in diesem Zusammenhang Otto von Gierke für den Modellcharakter des historischen deutschen Rechts in den Zeugensזand gerufen werden.

III.

Es gab also - das ist der Sinn des bisher Gesagten - gewichtige Gründe, welche die deurschrechtliche Wissenschaft zu einer Erneuerung ihres Selbstverständnisses veranlassen konnte, indem sie nämlich wiederum ihren Anteil an Rechtspolitik und Rechtsdogmatik verlangte. Die "Überfremdung" des deutschen Rechts konnte doch nur beseitigt werden, wenn man - wie es der Wiener Rechtshistoriker Rudolf Köstler ausdrüdkte - feststellte, w was am bisherigen Rechr deutsch ist $\alpha^{32}$, und das wiederum setzte die Kenntnis des alten Rechts voraus. Gewiß dachten auch die Bewunderer des mitrelalterlichen Rechts nicht daran, etwa den Sachsenspiegel als Gesetz einzuführen. Aber war es nicht an der Germanistik, die bestimmenden Strukturen des warteigenen $\propto$ Rechts aus dem von seiner zeitbedingten Gewandung befreiten germanischen und mittelalterlich-deutschen Recht zu entwickeln?

Es ist daher erstaunlich, wenn wir feststellen müssen, daB die deutsche Rechtsgeschichte weder zu einer grundsätzlichen Neuorientierung ihres Selbstverständnisses gelange ist, noch bei der *Rechtserneuerung « eine bestimmende Rolle gespielt har. Vielleicht weisen die internen Vorgänge in der Akademie für Deutsches Recht, die als *Zentralstelle für gesetzgeberische Vorarbeiten « eingerichtet war, manches interessante Detail auf; im Schriftum jedenfails sind nicht einmal deurliche Spuren eines Einflußkampfes zugunsten der Germanistik erkennbar ${ }^{33}$. Die Rechrsgeschichte blieb, wenn auch in der Studienordnung von 1935 reichlich mit Sternchenstunden bedacht ${ }^{34}$, weiterhin am Rande der Jurisprudenz; das historische Recht erhielt keine Wegweiserfunktion bei der Umwälzung des Rechts. Wenn ich den Gründen dafür nachgehe, so muß ich die politische Einstellung der Gelehrten außer Betrache lassen, da das vollständige biographische Material nicht leicht zu beschaffen wäre. Ich beschränke mich auf Ursachen, die im Charakter des Nationalsozialismus und den Bestrebungen der nationalsozialistischen Rechtswissenschaft und schließlich in der Rationalität der rechtsgeschichrlichen Forschung selbst begründer liegen.

Ein wirklicher Modellcharakter des historischen germanisch-deutschen Rechts, auch wenn er auf Grundlinien beschränkt gewesen wäre und den technisch-wirtschaftlichen Wandel berüdksichtigt hätte, widersprach eklatant dem Streben der Machthaber, rechtliche Festlegungen aller Art zu vermeiden, die das Regime selbst hätten begrenzen können ${ }^{35}$. Dieser Tendenz zufolge gale der Kampf des Nationalsozialismus dem $*$ formalen $\alpha$, will sagen dem fixierten und daher der Manipulation widerstrebenden Recht; an die Stelle des $\gg$ Formalrechts* sollte,

TI Was heiße und zu welchem Ende studicre man - Rechesgeschidite? ZAkDR, Jg. 1940, S. 194 ff. is Eine Kritik an Tendenzen, die bei der Arbeit am - Volkssesetzbuch der Deutschen sichtbar wurden, finder sich bei Franz Beyerle, Sduldenken und Geserzeskunst, Zeitschritt für die gesame Staatswissenschaft ro: (1942), S. 209. Beyerle warne insbesondere davor, das Ziel der Volkstümlichkeir aus den Augen zu verlieren.

34 Siche Karl August Eckhardt, Das Studium der Rechtswissenschaft, Hamburg 1935, S. 12. Bcsonders wichtige Vorlesungen wurden durch Sernchen gekennzeidiner.

${ }^{25}$ Dazu Rüthers, Die unbegrenzte Auslegung, S. $104 \mathrm{f}$. 
wie der $\gg$ Reichsrechtsführer « Frank es ausdrüdkres ${ }^{30}$, das „Lebensrecht * treten. - Recht und Unrechta, so heißt es bei Alfred Rosenberg ${ }^{37}$, wind nicht abstrakte Begriffe, nicht toter Buchstabe, sondern Recht ist das, was arische Menschen für Recht befinden, und Unrecht das, was sie verwerfen.« Freilich können auch geschichrliche Sachverhalte manipuliert werden, aber unter Wahrung rationalen Scheins nur bis zu einer bestimmten Grenze. Je konkreter sich der geschichtliche Stoff darstellt, desto eher ist diese Grenze ereicht.

Ein Beispiel: Die gängige Behauptung, das Führerprinzip sei germanischen Ursprungs und habe in der Herrschaft germanischer Stammesfürsten beispielhafte Verwirklichung gefunden, hatte für das Regime bei näherem Zusehen auch unangenehme Aspekte. Denn der germanische Herrscher war gerade nicht als Gesetzgeber begriffen, wo sich nicht kirchliche Einflüsse oder die Ubernahme spätrömischer Vorstellungen geltend machten. Eine Ubereinstimmung mit der nationalsozialistischen Rechtsquellenlehre, welche die Rechtsbildung dem Führertum in die Hand gab ${ }^{38}$, war daher nicht leicht herzustellen ${ }^{39}$. Das gleiche gilt für den rechtsgeschichtlichen Befund, wonach das echte deutsche Recht einer "künstlichen " Gesetzgebung abhold ist und sich durch organisches Wachstum auszeichner ${ }^{40}$. Auch im Privatrecht war die Festlegung durch das historische Argument keineswegs erwünscht. Kennzeichnend sind die Ausführungen, die der $*$ Reichsrechtsführer" Frank 1936 unter dem Thema "Eike von Repgow der Künder und Kämpfer des Rechts» gemacht hat. Er lobt den wwahrhaft deurschen Geist» des Sachsenspiegels, der durch die Grundwerte *Friede, Freiheit, Treue und Gefolgschaftspflicht « beherrscht sei, fährt dann aber fort:

- Es ist klar, daß die unmitteibare Obernahme der Vorschriften des mittelalterlichen Rechrs in unsere heurige Zeir nicht in Frage kommen kann *. Die moderne Rechtsordnung muß aber »in ihren Grundlagen ähnlich gefestigt und in ihren Zielen ebenso einheitlich ausgerichret sein, wic wir dies am Sachsenspiegel noch heure bewundern «41.

Einheitlichkeit und Geschlossenheit, Friede, Freiheit, Treue und Gefolgschaftspflicht, das sind die Schlagworte, auf deren historische Verzierung sich die rechesgeschichtliche Wissenschaft zu beschränken hat. Durch nichts anderes also konnte die Germanistik dem Regime nützen, als durch den Nachweis des genuin-germanischen Charakters einiger Parolen.

Ein dogmatischer Anspruch des historischen Rechts wurde ferner auch von der nationalsozialistischen Jurisprudenz keineswegs akzeptiert. Die Leitung der legislatorischen Vorarbeiten, die Führung in der Erneuerungsdiskussion wurden ganz überwiegend von Leuten übernommen, die entweder überhaupt nicht historisch oder nicht germanistisch orientiert waren und denen es ferne lag, ihre Arbeit mit einem engagierten Einstieg in die Rechtsgeschichte zu beginnen. Eine Gegnerschaft zur Renaissance der historischen Rechtsschule finden wir ausdrüdk-

\footnotetext{
so $\mathrm{DJZ} 1936$, S. 400 .

3: Das Reche wurzele im Volk, ZAkDR 1937, S. 610.

so Siehe Rüthers, Die unbegrenzte Auslegung, S. $127 \mathrm{ff}$.

so Einen Versuch dieser Art unternahm Georg Lenz, Die Erneuerung des Verfahrensrechis aus dem germanischen Rechtsdenken, Deutsche Rechtswissenschaft, Jg. 1937, S. 339 ff., der den Führer nicht als Gesetzgeber, sondern als den höchsten Urteilsfinder fungieren läßt: Aus naheliegenden Gründen komme eine direkte Befragung der Thinggenossen im heutigen Scaas niche in Betracht. Der Richter frage daher das Urteil an den Führer, der kraft seiner Stellung als einziger berufen ist, für die Gemeinschaft der Genossen zu sprechen und ihr Urteil zu finden «. Diese Urteilsfindung geschiche in einem Millionenvolk $\rightarrow$ in Form allgemeiner Aussprüdee (S. $3+0)$.

to In diesem Sinne z. B. Walther Merk, Wachstum und Sdöpfung im germanischen Reche, in: Beiträge zur Neugestaltung des Deutschen Rechts, Festgabe für Eridh Jung, Marburg 1937, S. 173.

41 Deutsches Recht, Jg. 1936, S. 298.
} 
lich bei Juristen, die von rechtsphilosophischen Ausgangspunkten her dem neuen Recht den Weg zu weisen versuchten. Die heutige Aufgabe der Rechtswissenschaft, schreibt Carl Schmitt im Jahre 1936, ist philosophischer Art ${ }^{42}$. Er streiter den Germanisten des 19. Jh. gewisse Verdienste nicht $a b$, leugnet aber, daß sie das eigentliche Problem gelöst hätten:

- Die Germanisten setzten den Normen und Begriffen einer romanistischen Jurisprudenz den Inhalt deutschrechticher Normen entgegen; mit gutem Recht und gutem Erfolg. Aber in der Hauptsache handelte es sich um mehr als um die Verschiedenheiten von Normeninhalten, über deren Richtigkeit und Zwedsmäßigkeit man streiten kann; der cigentliche Kampf ging um etwas anderes, nämlich darum, den Typus des rezeptionsrechtich geprägten Juristen durch den eines deurschen Rechrswahrers zu erseczen ....43

Das sei - so behauptet Carl Schmitt unter Hinweis darauf, daß es auch unter den Germanisten Juden gegeben habe, nicht gelungen. Entscheidend in diesem Zitat erscheint mir die Aussage, daß man über die Richtigkeit und $Z$ weckmäßigkeit des sog. Deutschen Rechts streiten könne; sein Inhalt entbehrt also jeglicher Vorbildlichkeit.

Letztlich - so meine ich - war jedoch die Wissenschaft vom historischen germanisch-deutschen Recht selbst weder willens noch in der Lage, in der Diskussion um die Rechtserneuerung die führende Rolle zu übernehmen. Trotz aller Rede von der Wiedererweckung des germanischen oder deutschen Geistes war auf wissenschaftlicher Ebene die Zeit romantischen Geschichtsdenkens vorbei, der Siegeszug der geschichtswissenschaftlichen Methode nicht revidierbar. Längst hatten Einzeluntersuchungen die Idee, man könne aus der Summe verstreut liegender partikularer Rechtsquellen ein Privatrechtssystem herleiten, erschüttert. An die Stelle historisch-orientierter Systematik war die Einzeluntersuchung getreten, welche die überkommene Gesamtschau im Detail korrigierte und damit insgesamt in Frage stellte. So erklärt es sich, daß gar nicht mehr der Versuch unternommen wurde, nach dem Vorbild Gierkes ein System des deutschen Privatrechts zu konzipieren ${ }^{44}$. Auch nach 1933 blieb die Forschung im Großen und Ganzen in den bisherigen Bahnen, ein Dogmatismus wird selten spürbar. Interessant erscheint in diesem Zusammenhang ein Blick auf die von dem Rechtshistoriker Karl August Eckbardt inspirierte Studienordnung von 1935 . Dort wird zwar für das erste Studiensemester eine 4- bis 6-stündige Vorlesung über »Germanische" oder "Deutsche« Rechtsgeschichte vorgesehen, wobei nach Möglichkeit die »Germanische« den Vorzug erhalten soll; die bisher übliche historisch-dogmatische Vorlesung indessen, genannt "Deutsches Privatrecht «, entfällt. Wenn es in der Studienordnung auch heißt, die "Germanische Rechtsgeschichte " solle auch die Entwicklung der einzelnen Rechtsinstitute umfassen, so ist dennoch die Entscheidung gegen die »Historische Rechtswissenschaft « und für eine Entwidklungsgeschichte des Rechts gefallen ${ }^{45}$.

Für einen Modellcharakter des historischen Rechts hätten sich zudem kaum überwindliche Schwierigkeiten ergeben. Die Quellen des vom römischen Recht nicht wesentlich erfaßten germanischen und deutschen Rechts liegen zeirlich über 1000 Jahre auseinander; sie stammen zudem aus höchst unterschiedlichen Kultursitua-

4: Die gesdridutlide Lage der deutsdaen Rechtswissenschaft, DJZ 1936 , S. 2 I.

13 Aufgabe und Norwendigkeit des deutschen Rechrsstandes, Deursches Recht, Jg. I936, S. I St; siche ferner Karl Larenz, Volksgeist und Recht, Zeitschrift für deutsche Kulturphilosophie, Jg. 1935, S. 40 ff.

44 Die *Grundzüge des deutschen Privatrechts* yon Rudolf Hübner, I. Aufl. 1908, s. Aufl. 1932, sind als Entwidklungsgeschichte konzipiert.

15 Siehe dazu Claudius von Scbwerin, Deutsdie Rechtswissenschaft, Jg. I939, S. I 88. 
tionen. Allein die Tatsache der germanischen Reichsgründung und der Christianisierung bedingten gewaltige Veränderungen des Rechts. Wo aber sollte man den für das historische Modell entscheidenden Querschnitt ansetzen? Sollte heidnisches oder von der Kirche beeinflußtes Recht die Richtschnur bilden? Die historische Orientierung, die noch beim Erbhofgesetz vom 29.9.1933 wenigstens in den Begründungen eine Rolle spielte, - es wurde die Wiederkehr des deutschen Eigentumbegriffs gefeiert ${ }^{46}$-, ist bereits beim Ehegesetz von $193^{8}$ nicht mehr zu spüren, und dies aus gutem Grund. Nationalsozialistisches Postulat war beispielsweise zwar niche die Gleichberechtigung, aber doch die *Höherwertung* der Frau. Hierfür konnte man freilich in den idealisierenden Berichten des Tacitus eine germanisch-historische Stütze finden; die germanischen Stammesrechte bieten jedoch ein davon völlig verschiedenes Bild: Die Frau erscheint, ehe der kirchliche Einfluß zum Tragen kommt, als bloßes Objekt der Eheschließung und steht unter der strengen Hausherrschaft der Männer. In der älteren Forschung hatten sich sogar Zweifel geregt, ob die Frau bei den Germanen nicht wie eine Sache behandelt worden sei. Die neueren Forschungen zeigten zwar ein etwas versöhnlicheres Bild von den familienrechtlichen Zuständen der Germanen, man sprach z. B. nicht mehr vom Frauenkauf als Eheschließungsform, sondern umschrieb den Vorgang durch andere Terminit7. Aber Bestimmungen wie die des angelsächsischen Rechts, wonach ein Ehebrecher verpflichtet wurde, auf eigene Kosten dem betrogenen Mann ein anderes Weib zu beschaffen, waren beispielsweise nicht zu übersehen ${ }^{48}$. Heidnisch-germanisct.es Eherecht kam also als Vorbild nicht in Betrachr, ebensowenig wie natürlich das kirchliche Ehemodell des Mirtelalters.

Einige bekannte Rechtshistoriker haben sich damals mit dem Verhältnis von Rechtsgeschichte und Gesetzgebung auseinandergesetzt. Auch hier spüren wir bei genauer Lektüre die Distanz, welche die rechtshistorische Forschung zu den Zeitproblemen gewinnen will. Interessant sind beispielsweise die Ausführungen, die Claudius von Sobwerin in einem I938 erschienenen Sammelband $*$ Zur Erneuerung des Bürgerlichen Rechts ${ }^{49}$ gemacht hat. Von Schwerin geht von der aktuellen Bedeutung der Rechtsgeschichte aus: Bei der Rechtserneuerung ist die Geschichte zu befragen. Der dogmatische Anspruch des historischen Rechts wird dann aber unter der präzisen Fragestellung, was denn die Rechtsgeschichte für die Geserzgebung leisten könne, erheblich reduziert.

- Dieser Neubau kann aber... niche etwa in der Weise entstehen, daß man heure Geltendes durch das ersetze, was in der Vergangenheir gegolten hat. Ewig ist der deursche Geist, aber zeirgebunden ist die Form, in der er sich verwirklicht, die einzelne Regel der Rechtsordnung, und ernsteste Prüfung schulden wir daher der Frage, inwieweir die einzelnen Formen früheren Rechts uns gemäß sind $* .30$

Daher hat die Rechtsgeschichte hauptsächlich die sgeistigen und sittlichen Grundlagen des germanischen und des deurschen Rechis « $^{31}$ klarzulegen, daneben kann sie auf Rechtssprache und rechtliche Ausdrucksform einwirken. Damit ist, so meine ich, eine Leerformel gefunden, mit deren Hilfe man die Bedeutung des

10 Vgl. die Beiträge zum Erbhofrechr in ZAkDR, Jg. 1935, S. 95 ז ff.

17 Zum gegenwärrigen Forschungsstand Paul Mikat, Arr. Ehe« in: Handwörterbuch zur deurschen Rechisgeschichte, Bd. I, Sp. 809 ff.

19 Aethelberht, c. 31 ; siehe Felix Lievermann, Die Gesetze der Angelsachsen, Bd. I, S. s (Nachdruck Aalen r 960$)$.

19 Schriften der Akademie für Deutsches Rechr, Gruppe Rechesgrundlagen und Rechrsphilosophie, Hert 7, München und Berlin 1939, S. 37 ff.

30 S. 39.

31 S. 41 . 
historischen Rechts programmatisch aufrechterhalten konnte, ohne praktische Folgerungen daraus ziehen zu müssen: der "Geist« des alten Rechts, oder - wie Walther Merk sagt $\rightarrow$ die Rechtsgesinnung ${ }^{32}$, und nicht das Recht selbst, ist in die Gegenwart cinzubringen.

Weiter noch geht Karl Gottfried Hugelmann, der sich mit der Forderung, das Kebsverhältnis wieder einzuführen, auseinandersetz ${ }^{53}$. Hugelmann wendet sich gegen die Methode, eine bestimmte altertümliche Epoche mit Modellcharakter zu versehen und fordert die Berücksichtigung der gesamten Geschichte. Auch wenn man Rechtsentwicklung als unglücklich, abwegig oder als Uberfremdung empfindet, so ist nach seiner Meinung eine sorgfültige Untersuchung angebrachr, "wieweit denn das so leicht vorschnell gefaßte Vorurteil wirklich zutrifft und ob nicht im Zuge der Entwicklung ohnedies der Volksgeist dasjenige, was an dem einmal Uberkommenen allzu fremd war, geändert bzw. ausgeschieden hat «. Hugelmann zieht sich schließlich auf die alte Betrachtungsweise der Geschichte als Lehrmeisterin $^{54}$ zurück und komme auf diesem Wege auch zur Beachtlichkeir des römischen Rechts.

Kehren wir, da Hugelmanns Position die eines Außenseiters ist, zu den Sätzen von Schwerins zurück. Auch sie befreien die rechtshistorische Forschung der Zeit von einem kaum tragbaren Ballast, nämlich der Notwendigkeit, ihre Fragestellungen permanent an akruellen Problemen auszurichten und in ihren Ergebnissen gegenwärtig "brauchbar« zu sein. Zwar akzeptiert auch von Schwerin die nationalsozialistische Forderung nach Politisierung der Wissenschaft ${ }^{\text {sz }}$, die so neu also nicht ist: Die Rechtsgeschichte muß, wie von Schwerin sagt, gegenwartsbezogen oder politisch betrieben werden ${ }^{56}$, aber er betont gleichzeitig, da $\mathbb{B}$ auch die sammelnde und feststellende, d.h. also die bloß von geschichtlicher Methode bestimmte Forschung nicht ohne Wert für die Rechtserneuerung sei. So ist auch die antiquarisch ausgerichtete Forschungstätigkeit gerecht fertigt. Der Forscher kann nach diesem Text also wählen, ob er sich an der Suche nach dem Geist des deutschgermanischen Rechts beteiligen und also seine Wissenschaft in den Dienst historischer Idcologieverzierung stellen oder ob er die Rationalität seiner Forschung wahren will.

Das Letzte ist nun ganz überwiegend, und zwar auch bei Forschern, die wir im Banne des Nationalsozialismus wähnen dürfen, geschehen. Ein Blick auf die Germanistische Abteilung der Zeitsctirift für Rechtsgeschichte beweist das in verblüffendem Ausmaß. Den Bänden ab 1933 ist - von Tagungsberichten abgesehen der politische Umschwung kaum anzumerken. Arr der Themenstellung, Merhode und Inhale der Aufsätze bleiben die gleichen wie in den Jahrgängen vorher. Wie auch im Großteil der monographischen Literatur werden konkrete historische Erscheinungen des Rechts untersucht, ganz überwiegend ohne den Drang zu

5: Wachstum und Schöpfung im germanischen Recht, S. 128. Auch Merk nimmt eine Stellung zwischen geschichtlicher Dogmatik und einer Distanzierung des alten vom gegenwärtigen Recht ein: - Das mittelalterlid-germanische Recht mit seiner Sdiöpferkraft und seinem Sinn für Unwägbarkeiten wird für uns immer das unerreichte Vorbild eines eigenwüchsigen und volkstümlidhen Rechts bleiben. Zwar ist es unmöglid, zeitbedingte Sätze und vergängliche äußere Erscheinungsformen unseres älteren Rechts künstlich wiederzubeleben ... Aber die Baugesinnung, die einst die Burgen und Dome des deutschen Mittelalters geschaften har, und die Rechtsgesinning und die Leitgedanken, welche das mittelalterliche germanische Recht beseelt und geiormt haben, können uns Maßstäbe für die Wertung der Gegenwart und Anregungen für die Gestaltung der deutschen Zukuntt geben *.

5. Rechtsgescicichte und Gesetzgebung, ZAkDR, Jg. $1939, \mathrm{~S} .676 \mathrm{f}$.

54 Dazu Reinhart Kosclleck, Historia Magistra Vitae, in: Natur und Geschidice, Karl Löwith zum 70. Geburtstag, Stuttgart-Bcrlin-Köln-Mainz 1968, S. $196 \mathrm{ff.}$

55 Für die Rechtswissensdıat: Frank, ZNkDR, 19;8, S. 459.

56 2. 2. O. S. 40. 
mühsam konstruierter Nutzanwendung für die Gegenwart. Die Rechtsgeschichte bewahrte so, wo sie nicht in mehr oder minder vagen Programmatiken den deutschen Geist beschwor, ihre Rationalität.

Das gleiche gilt im Ergebnis auch für die Romanistik, auf deren Lage ich in diesem Rahmen nicht näher eingehen kann. Da - wie gezeigt - das römische Recht weithin der Gegnerschaft der nationalsozialistischen Jurisprudenz ausgesetzt war und da es nur unter Aufgabe kritisch-wissenschaftlichen Denkens in die Ideologie des Nationalsozialismus einzubringen gewesen wäre, befand sich die Romanistik, obwohl von der wissenschaftlich konzipierten Studienordnung von 1935 in erheblichem Umfang berüdksichtigt, in der Defensive. Den Vorwürfen gegen das römische Recht wurde verschiedentlich durch Kontrastierung seiner Entwicklungsstufen entgegnet ${ }^{57}$. Im ganzen verstärkte sich der Trend weg von der römischen Rechtsdogmatik und hin zur antiken Rechtsgeschichte ${ }^{38}$. Freilich fand das römische Privatrechtssystem in Paul Koschaker einen bedeutenden und entschiedenen Verfechter. Dem völkischen Programm des Regimes zum Trotz forderte er vom Gedanken der Aufrechterhaltung der europäischen Kulturgemeinschaft aus die Wiedereinführung der systematisch-dogmatischen Vorlesung über römisches Privatrecht ${ }^{30}$ und versuchte, auch die Pandektistik des 19. Jh. gegen die üblichen Angriffe zu verteidigen ${ }^{80}$.

IV.

Soweit die wissenschattsgeschichtliche Skizze, deren Grundlinien - so ist meine bescheidene Hoffnung - auch in einer auf breitem Quellenmaterial basierenden Wissenschaftsgeschichte des Dritten Reiches nicht aufgegeben zu werden brauchen. Die Krise der rechtsgeschichtlichen Wissenschaft als Teil der Jurisprudenz und als Element der Juristenausbildung besteht - und zwar, wie ich zu zeigen versuchte, im wesentichen ununterbrochen - seit Beginn des Jahrhunderts bis heute. Die Krise bedeutet zugleich eine Chance. Ein Zeitgeist, dem die historische Auslotung seiner Seinsbedingungen so fern steht, wie dies bei dem unsrigen der Fall ist, befrachtet die Geschichte nicht mit dogmatischen Aufgaben; die in unserer Gesellschaft konkurrierenden Sozialmodelle beziehen zumeist ihren utopischen Charakter nicht aus der Geschichte. Damit sind für die Geschichtsforschung die Versuchungen geringer geworden, irrational zu werden. Auch ich bin freilich der Auffassung, daß die Geisteswissenschaften von persönlichen Grundwertungen aus betrieben werden, die sich in einem letzten Kern der Durchleuchtung durch den kritischen Verstand entziehen werden. Es wäre aber falsch, deshalb vor dem Irrationalismus sogleich das Handtuch zu werfen und aus dem Mangel eine Tugend zu machen. Eine Recheswissenschaft, die lediglich der juristischen Verfesti-

57 Vgl. vor allem Max Kaser, Rötnisches Recht als Gemeinsdaftsordnung, Tübingen 1939. Den Unterschied zwishen dem antik-römishen Recht und dem der neveren Pandekristik stellen heraus z. B. Franz Wicacker, Die Stellung der römischen Rechrsgeschichte in der heutigen Rechtsausbildung, ZAkDR J5. 1939. S. 403 ff.; Hans Kreller. Die Bedeutung des römischen Rechts im neuen deutschen Hodschulunterriche, ZAkDR Jg. 1936, S. 409. Eine Deutung des römischen Rechts unter Zuhilfenahme nationalsozialistischer Modellbegriffe unternahm Ernst Sóönbauer, siehe bei Kaser 2. a. O., S. 7 f.

53 Vgl. Wieceler a. a. O.; Eriós Genzmer, Was heißt und zu welchem Ende studiert man antike Rechesgeschichie?, ZAkDR J5. 1936, S. 403 ff.

59 Pail Kosdjaker, Die Krise des römischen Rechis und die romanistische Recheswissenschaft, München-Berlin 1938; Probleme der heutigen romanistischen Rechtswissenschaf, Deutsche Rechrswissenschaft, Jg. 1940, S. I I o ff.

6. Die Ǩrise des römischen Rechrs und die romanistische Recheswissenschaft, S. 33. 
gung vorgefertigter Dogmen, Sozialmodelle oder akzeptierter Grundideologien dient, wäre ein jämmerliches Geschüft ebenso wie eine Geschichtswissenschaft, die sich unter dem Schlagwort der ${ }^{2}$ Politisierung * dazu hergäbe, sich in der Kunst der Ideologieverzierung zu üben. Dem Wissenschaftler bleibt die entsagungsvolle Aufgabe, auch seine Grundwertungen und Standorte vor das Gericht der kritischen Vernunft zu laden, soweit er das in der Bedingtheit seines Denkens nur immer vermag.

Die rechtsgeschichtiche Wissenschaft wird dann cine wesencliche Aufgabe in der Rechtswissenschaft behalten können, wenn sie sich die Aufgabe stellt, die Relativität jedweden Rechts, seine Gestaltungsmöglichkeiten, seine Erscheinungsformen, die es bestimmenden Faktoren, seine Abhängigkeit von der Kultursituation transparent zu machen. So wird sie mit allen Vorteilen einer Erfahrungswissenschaft zum rechtlichen Grundproblem beitragen, nämlich der Frage, was Recht ist und sein soll, und welche Seinsweisen des Reches in der menschlichen Existenz beschlossen liegen. 